\title{
Proposal of Automatic Ventilation Support System Utilizing Carbon Dioxide Concentration
}

\author{
Toshimasa Noda ${ }^{\text {a }}$ Yuhki Kitazono ${ }^{\mathrm{a}, *}$

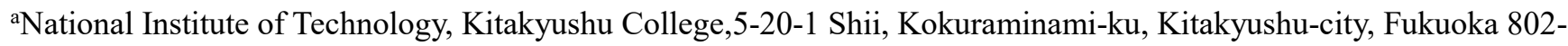 \\ 0985, Japan \\ *Corresponding Author: kitazono@kct.ac.jp
}

\begin{abstract}
The new coronavirus is prevalent, and importance of ventilation is exclained due to it. In such urgent situation, we propose a ventilation support system that uses carbon dioxide sensors and wind sensors more efficiently than conventional products. If ventilation is insufficient, concentration of carbon dioxide will increase, so if being insufficient, the window will automatically open. We will install a wind sensor in the place where the window is open, and check if ventilation is available there. If there is no ventilation, it helps ventilate by spinning the fan and generating an air current. The proper ventilation assistance was confirmed an experiment we made.
\end{abstract}

Keywords: COVID-19, Ventilation, Carbon Dioxide, Wind, Servo Motor.

\section{Introduction}

In December 2019 an outbreak of coronavirus disease 2019 (COVID-19) occurred in Wuhan ${ }^{(1)}$. The new virus is prevalent all over the world. Infection of the new coronavirus is mainly caused by coughing and sneezing of the virus holder, and is caused by droplets inhaled from the nose or mouth by others ${ }^{(2)}$. Furthermore, there is a possibility of infection from smaller particles droplets generated through conversation, or by touching the mucous membrane of the nose, mouth, eye, etc., of an infected person with a viruscontaining droplet or by touching the body. Recommended prevention from such new coronavirus include keeping a safe distance, washing hands frequently, and ventilation. This time, we focused on ventilation, which is important for preventing infection. According to the standard of ventilation provided by the Ministry of Health, Labour and Welfare, the number of ventilation times must be at least 2 per hour. If there are multiple windows to create air flow, you should open the windows on the two-way wall, if only one window, open the door. Concretely, the content of carbon monoxide in the air is $\leq 10 \mathrm{ppm}$ and the content of carbon dioxide is $\leq 1,000 \mathrm{ppm}$. Airflow shall be at least $0.5 \mathrm{~m} / \mathrm{s}^{(3)}$. These conditions are the criteria for ventilation. Of course, if you can keep the windows open or ventilate for a long time, but the effect of air conditioning and heating will. Hence, we wanted to develop a device that could detect the amount of carbon dioxide in the air and the flow of air currents and only ventilate as necessary. There are already machines that detect carbon dioxide. The machine tells us when the concentration of carbon dioxide reaches a certain level. However, subsequent work must be done by a person.

An automatic ventilation support system is proposed measure carbon dioxide concentration, automatically open windows, detect wind power, and send air through fans if there is not enough wind.

\section{Automatic Ventilation Support System}

Figure 1 shows the automatic ventilation support system created by this study. A carbon dioxide sensor and a wind power sensor are connected to Arduino and can be detected in order to add a function to automatically open the window as well as the concentration of carbon dioxide. In this system, the conditions are divided into three according to the concentration of carbon dioxide and wind speed. The first condition is that the concentration of carbon dioxide is below $1,000 \mathrm{ppm}$ and no ventilation is required. This state is defined as A. The second condition is that the concentration of carbon dioxide exceeds $1,000 \mathrm{ppm}$ and ventilates at wind speeds of $0.5 \mathrm{~m} / \mathrm{s}$ or more. This state is defined as B. In 


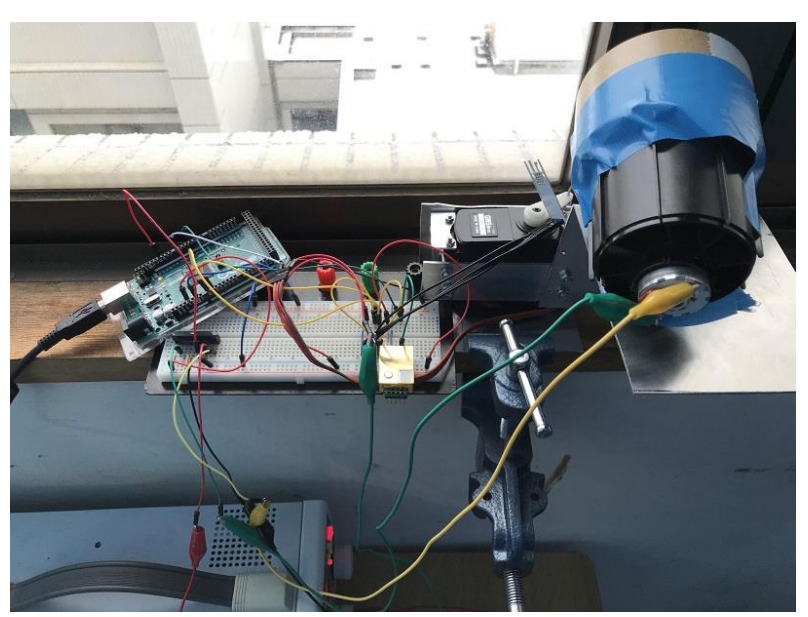

Fig. 1. A general view.

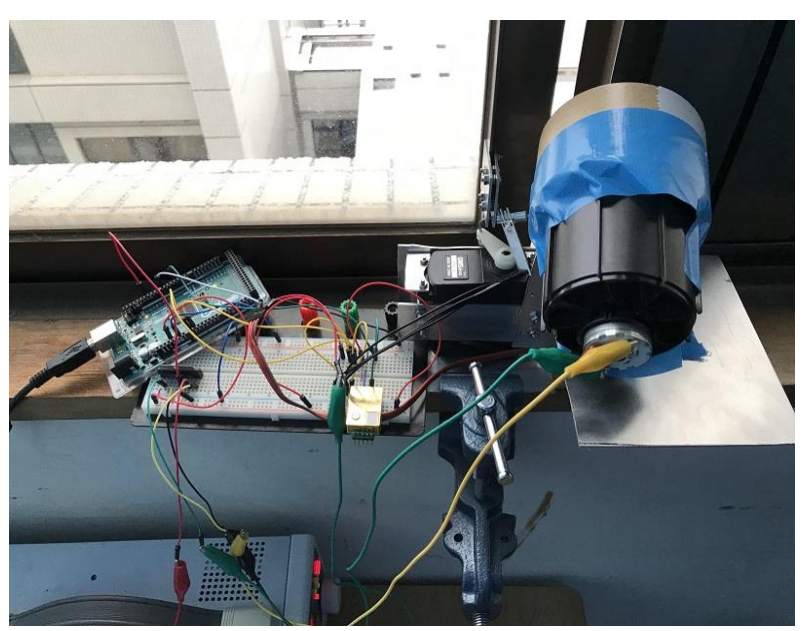

Fig. 2. A general view (window open).

addition to the $B$ condition, the wind speed is below $0.5 \mathrm{~m} / \mathrm{s}$, and the fan is turned to send air. This state is defined as C. In the case of $\mathrm{A}$, if concentration carbon dioxide goes beyond $1,000 \mathrm{ppm}$ for one minute, the A state ends and the window is moved to B state. In the case of B state, as shown in Fig. 2 , when the carbon dioxide concentration continues below $600 \mathrm{ppm}$, the servo motor is turned and the window is returned to A state. When the wind speed is below $0.5 \mathrm{~m} / \mathrm{s}$ in B state, the fan is switched to $\mathrm{C}$ state. After that, when the carbon dioxide concentration drops below 600ppm, the fan is closed with a servomotor. After the window is closed, the fan is returned to A state.

\subsection{Carbon dioxide}

For the carbon dioxide sensor MH-Z19B was used. Carbon dioxide absorbs infrared rays of 4,260 $\mathrm{nm}$. This carbon dioxide sensor can measure carbon dioxide concentration from $0 \mathrm{ppm}$ to $2,000 \mathrm{ppm}$. It is controlled by Arduino to keep measuring room concentration. When it exceeds $1,000 \mathrm{ppm}$, the window signals to the servomotor.

\subsection{Wind power}

Wind Sensor Rev. C was used as the wind force sensor. This wind force sensor is a thermal anemometer based on a conventional technique for measuring wind speed called "heat ray" technology. The wind speed is proportional to the heat, or the power, applied to the sensor. Heat ray technology is superior in low to moderate wind speed, so this sensor was used.

\subsection{Window}

A system is installed at a window side part to open and close the window as shown in Fig. 1. The windows were opened and closed with wires attached to the servomotor. For the servomotor GWServo's s666N was used. The width of the windows was approximately 2 meters long.

\subsection{Fan}

For the fan, the fan attached to the dryer of the DRRM66E2 is used removed from the dryer. Add 18 volts to this to generate wind. The fan motor driver controls it. We used TA7291P.

\section{Experiment}

In the experiment, the size of the classroom, which is regulated for school, is set to be 74 square meters by the Ministry of Education, Culture, Sports, Science and Technology ${ }^{(4)}$. Each class has a capacity of 35 students, so the area per person is approximately 2.1 square meters. In this experiment, a sealed space of about 2.1 square meters was created. We went in there alone and took measurements.

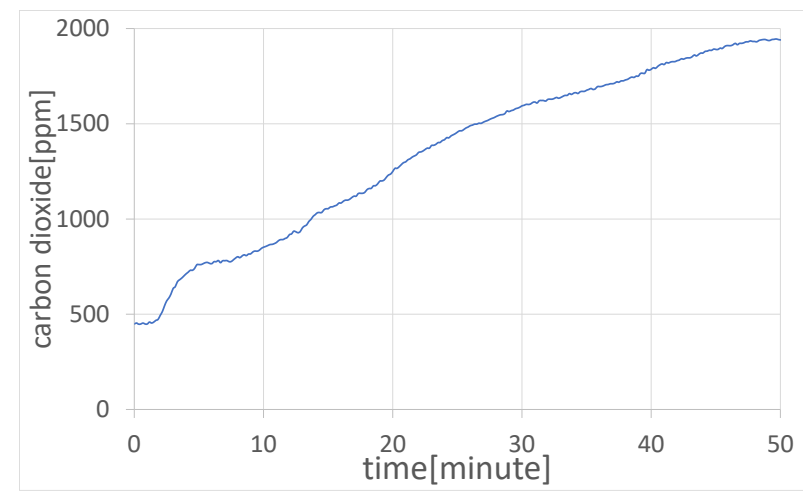

Fig. 3. The rate of increase in carbon dioxide. 


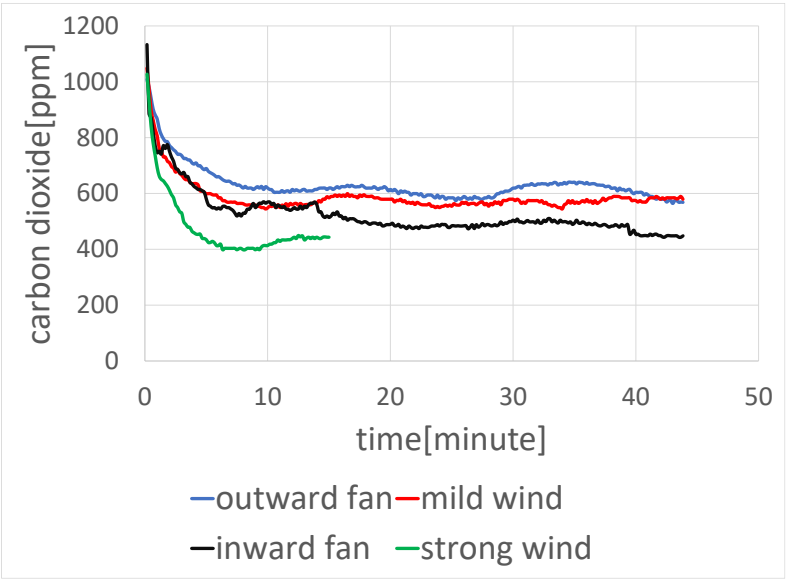

Fig. 4. Four patterns of carbon dioxide concentration.

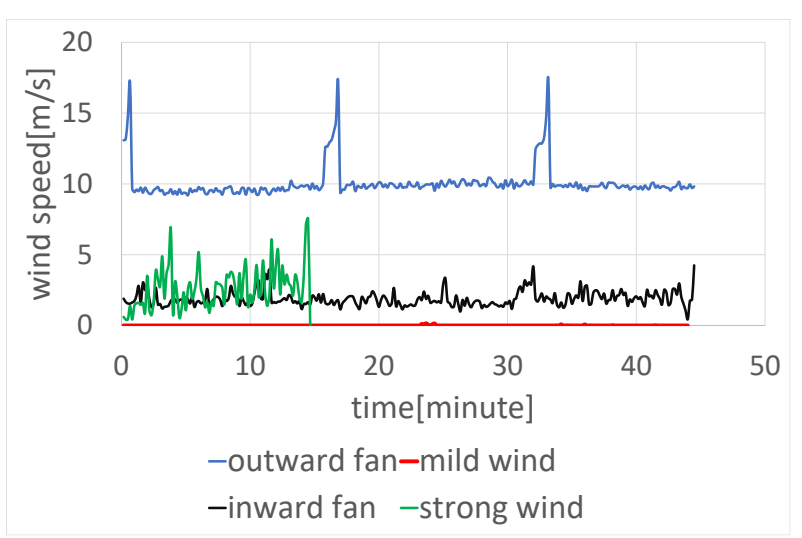

Fig.5. Four patterns of wind speed.

\subsection{The rate of increase in the concentration of carbon dioxide in a closed room}

An experiment was conducted to measure how carbon dioxide concentration increases in one class. In 50 minutes per lesson, we measured how high the concentration would increase during that time. The result is shown in Fig. 3. As you can see in Fig. 3, if the window is not opened for 50 minutes, it will exceed the standard value of $1,000 \mathrm{ppm}$.

\subsection{Fan availability changes time during ventilation}

Some experiments were conducted on how carbon dioxide levels fell when the window was opened to 1,000 ppm or more. First, when the window was opened, and when the wind was weak, ventilation was supported with the fan. The fan recorded both inward and outward. Finally, on a windy day. The four changes in carbon dioxide concentration and wind speed are shown in Figs. 4 and 5.

(1) Weak windy days

We conducted an experiment on a windless day. The carbon dioxide concentration was around $600 \mathrm{ppm}$ when the window was opened, so we conducted an experiment to help

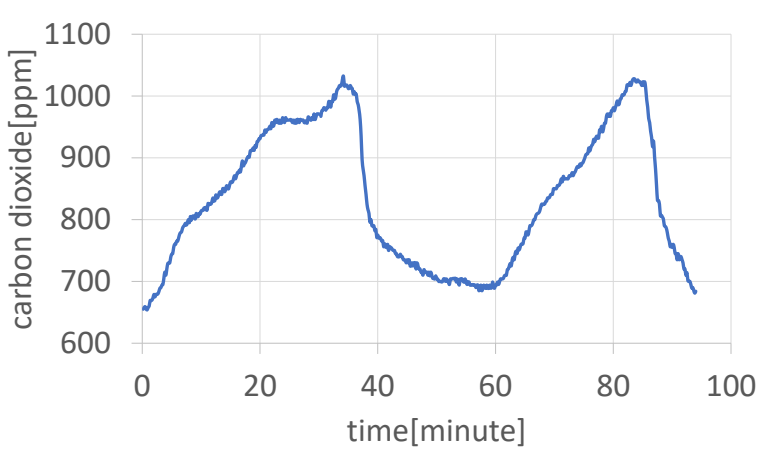

Fig. 6. System verification (carbon dioxide).

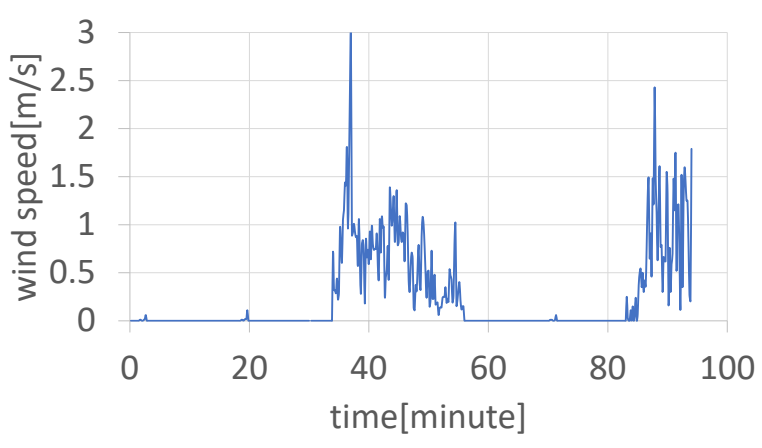

Fig. 7. System verification (wind speed).

ventilate with the fans. The fans did both outward and inward. When the fan was turned outward, the value was almost the same as without the fan, perhaps negating the incoming wind. When the fan was turned inward, the price went down.

(2) Ventilation on windy days

The window was opened on a windy day. On a windy day, the ventilation was so smooth that no assistance from the fans was needed.

\subsection{Experiment with this system}

Finally, an experiment was conducted to verify the overall operation of the system. Figure 6 shows the changes in the concentration of carbon dioxide in this experiment. Figure 7 shows the wind speed transition of this experiment. The experiment opened the door to a simulated room made in 3.1. The size of the window is $1 \mathrm{~m}$ long and $5 \mathrm{~cm}$ wide. How can the window be opened automatically when the carbon dioxide concentration rises? How does the fan run normally? We could see the fans moving when the wind speed was low. Also, it was confirmed that the window was closed automatically when the value below $600 \mathrm{ppm}$ continued for one minute. This showed that the system was working as expected. 


\section{Conclusions}

In this study, we proposed an automatic ventilation system using the concentration of carbon dioxide. The system succeeded in keeping the concentration of carbon dioxide below a certain level by opening and closing windows. As issues to be tackled in the future, it can be considered that the window can be opened widely, and that ventilation can be performed even in one direction.

\section{References}

(1) Kenji Karako, Peipei Song, Yu Chen, Wei Tang, "Analysis of COVID-19 infection spread in Japan based on stochastictransition model", BioScience Trends. 2020; 14(2):134-138

(2) World Health Organization, Natural ventilation for infection control in health-care settings, WHO guidelines 2009

(3) Ministry of Health, Labour and Welfare, $\sim$ To all managers of commercial facilities, etc. $\sim\ulcorner$ an enclosed space with poor ventilation $」$ ventilation methods for improving.(https://www.mhlw.go.jp/content/10900000/ 000618969.pdf)

(4) Ministry of Education, Culture, Sports, Science and Technology, Regarding the deliberation of the systematic development of detailed leadership system by a small number of people, 2020/10/21 